\title{
Malignant melanoma of the right atrium: antemortem diagnosis by transvenous biopsy
}

\author{
JAYAKRISHNAN A GOSALAKKAL, * DECLAN D SUGRUE $†$ \\ From the Departments of ${ }^{\star}$ Cardiothoracic Surgery and $†$ Cardiology, Mater Misericordiae Hospital, Dublin, \\ Republic of Ireland
}

SUMMARY About half of all patients with malignant melanoma have cardiac metastases. Histological confirmation antemortem of a secondary malignant melanoma of the heart is difficult. The diagnosis of a secondary melanoma in the right atrium was confirmed by transvenous biopsy in a 46 year old woman who had had a melanoma excised from her right leg two years before.

Invasion of the heart by a secondary tumour is much more common than a primary cardiac neoplasm. ${ }^{1}$ Nearly half of all patients with malignant melanomas have cardiac metastases. ${ }^{2}$ Histological antemortemconfirmation of secondary tumours of the heart is difficult and to our knowledge diagnosis of a secondary melanoma of the heart by transvenous biopsy has not been reported before. We report one such case.

\section{Case report}

A 46 year old woman was referred with influenza like symptoms and a systolic murmur. She had had a melanoma excised from her right leg two years before.

Physical examination was normal apart from a soft ejection systolic murmur at the left sternal border and a tumour plop. Full blood count, liver function tests, and electrocardiogram were normal. Chest $x$ ray showed a small opacity in the right lower lobe. A computed tomographic scan of the thorax showed a small solitary pulmonary nodule in the right lower lobe. The right atrium was incidentally noted to be enlarged and contained several areas of low attenuation suggesting a tumour within the chamber.

Cross sectional echocardiography showed a mass in the right atrium. The exact attachments were uncertain but it seemed to be attached to the interatrial septum. The mass slightly displaced the tricuspid valve and prevented leaflet apposition in systole (figure). Colour flow Doppler showed slight tricuspid regurgitation. Right ventricular function was normal.

Requests for reprints to Mr Jayakrishnan A Gosalakkal, Department of Cardiothoracic Surgery, Mater Misericordiae Hospital, Eccles Street, Dublin 7, Republic of Ireland.

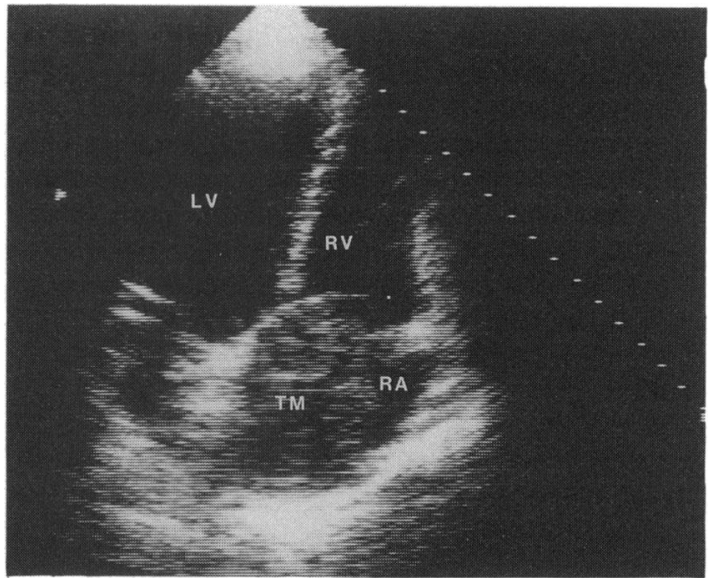

Figure Cross sectional echocardiogram showing tumour mass (TM) in the right atrium, impinging upon the tricuspid valve. $L V$, left ventricle; $R V$, right ventricle; $R A$, right atrium.

Cineangiography with contrast injection into the superior vena cava confirmed the presence of a large filling defect in the right atrium with slight dilatation of the superior vena cava. A transvenous biopsy of the right atrial mass was performed via the right internal jugular vein. Histological examination showed that the tumour was composed of a monomorphous population of polygonal cells containing large vesicular nuclei, prominent nucleoli, and abundant eosinonophilic cytoplasm. No melanin was identifiable. Immunocytochemistry showed the tumour cells to be strongly positive for $\mathrm{S} 100$ and vimentin, which confirmed that it was a malignant melanoma. 


\section{Discussion}

Space occupying tumours of cardiac chambers can give rise to signs and symptoms that are indistinguishable from those of other forms of myocardial or valvar heart disease. ${ }^{3}$ Diagnosis is facilitated by a high degree of clinical suspicion. In the present case, diagnosis was established by a combination of echocardiography and cineangiography and confirmed by transvenous biopsy. This report shows that transvenous biopsy of intracavitary cardiac neo- plasms is practical. However, samples may be small and histogenesis not always easily ascertained.

\section{References}

1 Harvey WP. Clinical aspects of cardiac tumours. Am J Cardiol 1968;21:328-43.

2 Thomas JH, Panoussopoulos DG, Jewel WR, Pierce GE. Tricuspid stenosis secondary to metastatic melanoma. Cancer 1977;39:1732-7.

3 Sterns LP, Eliot RS, Varco RL, Edwards JE. Intracavitary cardiac neoplasms. A review of fifteen cases. Br Heart J 1966;28:75-83. 